\title{
Model-based analysis and optimization of the mapping of cortical sources in the spontaneous scalp EEG
}

\author{
ANDREI V. SAZONOV $\uparrow * *$ JAN W. M. BERGMANS $\uparrow$, PIERRE J. M. CLUITMANS $\uparrow$, \\ PAUL A. M. GRIEP $\ddagger$ JOHAN B. A. M. ARENDS $\ddagger$ and PAUL A. J. M. BOON $\$ \S$ \\ $\dagger$ Signal Processing Systems Group, Department of Electrical Engineering, Eindhoven University of Technology, \\ Eindhoven, The Netherlands \\ $\ddagger$ Department of Clinical Neurophysiology, Epilepsy Center Kempenhaeghe, Heeze, The Netherlands \\ I Center for Electrophysiological Diagnostics, Vlijmen, The Netherlands \\ $\S$ Laboratory for Clinical and Experimental Neurophysiology (LCEN), Department of Neurology, Reference Centre \\ for Refractory Epilepsy, Ghent University Hospital, Ghent, Belgium
}

(Received 19 March 2007; revised 13 June 2007; in final form 20 June 2007)

\begin{abstract}
The mapping of brain sources into the scalp electroencephalogram (EEG) depends on volume conduction properties of the head and on an electrode montage involving a reference. Mathematically, this source mapping (SM) is fully determined by an observation function (OF) matrix. This paper analyses the OF-matrix for a generation model for the desynchronized spontaneous EEG. The model involves a four-shell spherical volume conductor containing dipolar sources that are mutually uncorrelated so as to reflect the desynchronized EEG. The reference is optimized in order to minimize the impact in the SM of the sources located distant from the electrodes. The resulting reference is called the localized reference (LR). The OF-matrix is analyzed in terms of the relative power contribution of the sources and the cross-channel correlation coefficient for five existing references as well as for the LR. It is found that the Hjorth Laplacian reference is a fair approximation of the LR, and thus is close to optimum for practical intents and purposes. The other references have a significantly poorer performance. Furthermore, the OF-matrix is analyzed for limits to the spatial resolution for the EEG. These are estimated to be around $2 \mathrm{~cm}$.
\end{abstract}

Keywords: Model; EEG; Reference; Optimization; Spatial resolution; Localization

\section{Introduction}

The scalp electroencephalogram (EEG) is a record of fluctuations of electrical activity in the brain recorded from the surface of the scalp. The records are based on the ability of neurons to generate current flows in surrounding biological tissues. The tissues are called the volume conductor (VC). The current flows across the VC produce potential changes that can be detected from the surface of the scalp [1].

Essential requirements for a significant potential change on the scalp are spatial organization and synchrony of neurons [2]. In this work, we restrict ourselves to neuronal populations located in small columnar cortical volumes, called cortical macrocolumns [3].

*Corresponding author. Email: a.sazonov@tue.nl

Computational and Mathematical Methods in Medicine 
Within the macrocolumns, neurons are highly correlated in their discharging time and have similar orientations.

At a distance, each macrocolumn can be approximated by an equivalent source of a signal. At each place on the scalp, the electrical potential is a linear mixture of all the source signals. The mixture depends on the location on the scalp relative to the sources and on the VC [4].

The EEG recording procedure requires an electrode montage which involves an electrode array and a reference [1]. The reference defines how the reference potential is obtained. It can be a potential at one of the electrodes or a linear combination of the potentials at several electrodes. Any EEG channel is recorded as a difference between the potential at the recording electrode and the reference potential. This way of mapping the potentials into the EEG channels is called the referencing procedure. This procedure is not invertible in the sense that it is impossible to reconstruct the potentials at each of the electrodes. However, it is possible to re-reference the EEG, i.e. to switch from one reference to another and vice versa (the re-referencing procedure).

The properties of the $\mathrm{VC}$ and the choice of the reference together determine the mapping of the cortical sources into the EEG channels (i.e. the source mapping (SM)). Whereas the contribution of the VC is fixed, the reference can be selected. For instance, a human expert may re-reference the EEG while inspecting it visually for a "better" representation of the brain activity.

Numerous clinical applications require accurate estimates of electrical activities of distinct cortical areas. Therefore, a reference that provides such estimates is essential. It should be noted, however, that for the related but different problem of source localization the reference is of a minor importance [5].

Although the importance of the SM is generally known, it remains under-exposed, and is underestimated or even neglected in numerous studies. The SM is essential for visual inspection as well as for quantitative EEG analysis, and has to be considered in order to avoid misinterpretations of EEGs. For instance, the choice of the reference affects the EEG by spatial filtering and dispersion of the source signals across the EEG channels [6], and therefore may significantly influence results of EEG analysis.

It is difficult to determine the SM for the head of a person since the activities and locations of all the sources are unknown and cannot be measured. Nevertheless, available physiological knowledge allows building models which can support objective analysis of the SM. The SM was partly investigated in a few papers. Nunez et al. [6] and Srinivasan et al. [7] investigated the SM in terms of the coherence function of the EEG channels, computed for different references. They used a three-shell spherical head model containing radial sources in the inner sphere. The effects of different references on the coherence function were discussed in terms of simulations with artificial EEGs. The main conclusion was that the choice of the reference introduces unique spatial filtering of source activity.

It was found that the Laplace operator of the scalp potential reflects the radial current density across the skull, and thus can be associated with primary activities originating within local areas under the electrodes. Based on this foundation, Hjorth proposed a reference which provides a finite central difference approximation of the scalp Laplacian [8]. Later, many other approximations of the surface Laplacian were proposed. Most of them are based on spline interpolations of recorded scalp potentials. Although these approximations are, in general, more accurate than the Hjorth Laplacian reference (HR), they depend on the choice of a spline function, and require a priori knowledge on head geometry, exact electrode locations and sophisticated computations [7,9,10]. Furthermore, since the spline-based 
interpolations depend on stochastic properties of the potentials, the references corresponding to the interpolations are time-variant.

The main goal of this paper is to analyze the SM for existing references, and to optimize the reference so as to minimize the impact in the SM of the sources located distant from the electrodes. We analyse the SM for the references that are independent of scalp potentials and, thus, are fully determined for a given VC and an electrode montage.

We introduce a time-invariant generation model for the desynchronized spontaneous EEG. This model is used to analyze and to optimize the reference. The model consists of three parts: (1) sources, (2) volume conductor and (3) electrode montage. The first two parts model the head and the third part models the EEG recording procedure; see figure 1. The sources are uncorrelated, thus mimicking the desynchronized spontaneous EEG. They are associated with cortical macrocolumns and modeled by dipoles which have orientations [3]. The montage involves 19 basic electrodes of the standard 10-20 electrode system, two mastoid electrodes, and one of the six references: ideal silent, common electrode, common digitally linked mastoids, common average, HR and the novel LR. The ideal silent reference is not physically realizable but included for comparison. We proceed as follows.

Firstly, we mathematically formalize the SM in the form of the observation function (OF) matrix, which defines the contribution of each source in each EEG channel. The OF-matrix can be explained as a product of the forward matrix and the reference matrix, corresponding to the VC and the reference respectively.

Secondly, we optimize the reference using the OF-matrix for the model. The reference is optimized to minimize the impact in the SM of the sources located distant from the electrodes. This optimisation leads to a new reference which we call the localized reference (LR). The LR provides "focused" estimates of the activities under the electrodes and separates these activities in different EEG channels.

Thirdly, we analyze the OF-matrix in order to assess the performance of different references for estimation of activity of the sources located close to the electrodes. The performance measures used for the analysis are the relative power contribution (RPC) of sources having different distances from the electrode, and the cross-channel correlation coefficient (CCC). Both measures are computed from the OF-matrix and are independent of the stochastic properties of the sources. The results are presented for the existing references as well as for the LR. It is found that the LR can be regarded as a refinement of the HR (up to $15-20 \%$ better performance), and that both of them have significantly better performance

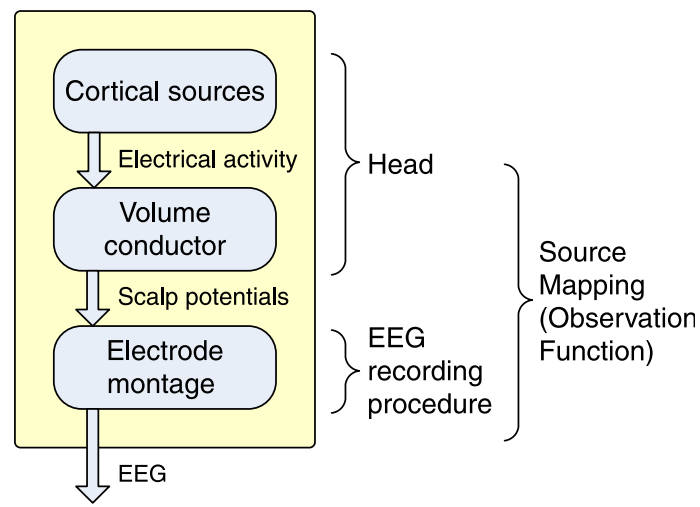

Figure 1. A block diagram for the generation model for the desynchronized spontaneous EEG. 
compared to the other references. We conclude that the LR is a fair alternative to the HR for recording of local cortical activities in different EEG channels especially when a head model is available. We note, however, that the HR is found to be close to the LR, and thus is close to optimum for practical intents and purposes.

Finally, we show that model errors (e.g. fluctuations of the VC properties) can cause significant errors in the EEG channels. The errors in the EEG channels depend heavily on the density of the electrode array, and are substantial for arrays with more than 140 electrodes, or equivalently, for inter-electrode distances below about $2 \mathrm{~cm}$.

\section{Generation model for the desynchronized spontaneous EEG}

\subsection{Volume conduction part}

The volume conduction part of the model is based on the classical spherical head model introduced by Rush and Driscoll [11]. Our modification consists of four concentric spherical layers corresponding to the brain, dura, skull and skin. Each layer $i$ is defined by its outer radius $R_{i}=\{80,82,87,92\} \mathrm{mm}$ and conductivity $\sigma_{i} \in\{0.33,1.0,0.0042,0.33\} \mathrm{S} / \mathrm{m}$. These values are taken from other studies and correspond to the head of an average adult [7,12]. Recently, other values were reported for the conductivity of the skull, see e.g. [13] and references therein. However, the results show no critical dependence on $\sigma_{i}$ and therefore we prefer to use the classical values.

The four-shell spherical VC is an approximation (within 10-20\%) of more complex and more realistic models such as an ellipsoidal shell model [14] and a finite-element model [15]. For our purpose, this accuracy is sufficient since we study general phenomena rather than, e.g. the exact source location in a particular head.

\subsection{Source part}

The human cortex consists of roughly $10^{10}$ neuronal cells having multiple synaptic connections each of which may serve as a source of electrical field. To model this complex system, we reduce the amount of functionally relevant units. The cortex is modeled as a set of cortical macrocolumns which are small cortical volumes containing many neurons. At a distance, each macrocolumn may be regarded as a single source of electrical field, which can be approximated by an equivalent electrical dipole [3]. Each dipole has a fixed location in the cortical layer, a fixed orientation and a variable strength, which defines the force of the field.

This strength is modulated by a white noise signal with $\mu=0$ and $\sigma^{2}=1$. For different dipoles, the noise signals are mutually uncorrelated. Hence, the dipoles can be regarded as generators of the desynchronized EEG. This is an important type of EEG which was simulated earlier using similar models [3,6,7]. In many practical situations, EEGs can be approximated as desynchronized even if the sources are weakly correlated [16].

The dipoles are oriented radially with respect to the spheres of the VC, which corresponds to preferred orientations of the macrocolumns. Moreover, the dipoles are uniformly distributed in a segment of the surface of a concentric sphere having $78 \mathrm{~mm}$ radius. This segment is constrained in polar coordinates by a maximal zenith angle of $105^{\circ}$ (with respect to the location $\mathrm{C} z$ ) that corresponds to the approximate location of the cortex. Based on physiological properties of the cortex, the upper bound of the total number of dipoles is estimated at $\sim 10^{4}$, which is the number of macrocolumns [3]. However, we will justify 
in section 5.3 that this number can be reduced to $10^{3}$ without significant performance changes for the model.

\subsection{Recording montage}

For the majority of clinical applications, the electrode array is limited to the standard 10-20 system having a typical interelectrode distance about $5 \mathrm{~cm}$ [17]. Since this system is most widely used in clinical practice, it is utilized for the model. For practical purposes, the reference electrodes A1 and A2 are replaced by two mastoid reference electrodes M1 and $\mathrm{M} 2$.

We analyze and compare the results for the ideal silent reference, four existing conventional references, and the novel LR. The existing references are: common single mastoid electrode, common digitally linked mastoids, common average and the HR. They are generally accepted and widely used in clinical practice for visual inspection as well as for quantitative EEG analysis $[1,6]$.

\section{Mathematical description and performance measures}

\subsection{OF-matrix}

The EEG channels are recorded as differences between electrode and reference potentials. These potentials are induced by brain sources. Let $M, L$ and $K$ be the total numbers of sources, electrodes and EEG channels respectively. The corresponding signals are denoted by vectors $\vec{s}=\left[s_{m}\right]$ of size $1 \times M, \vec{p}=\left[p_{l}\right]$ of size $1 \times L$ and $\vec{c}=\left[c_{k}\right]$ of size $1 \times K$.

The mapping of the source signals through the scalp potentials into the EEG channels (i.e. the $\mathrm{SM}$ ) is determined by the system:

$$
\left\{\begin{array}{l}
\vec{p}=\vec{s} \mathbf{W} ; \\
\vec{c}=\vec{p} \mathbf{R},
\end{array}\right.
$$

where the matrices $\mathbf{W}=\left[w_{m l}\right]$ of size $M \times L$ and $\mathbf{R}=\left[r_{l k}\right]$ of size $L \times K$ determine, respectively, the mapping of the source signals into the scalp potentials, and the mapping of the scalp potentials into the EEG channels. Hence, the matrices $\mathbf{W}$ and $\mathbf{R}$ characterize the VC and referencing effects respectively. The matrix $\mathbf{W}$ is called the forward matrix and $\mathbf{R}$ is called the reference matrix.

The equations of system (1) can be combined as

$$
\vec{c}=\vec{s} \mathbf{W} \mathbf{R}=\vec{s} \mathbf{Z},
$$

where $\mathbf{Z}=\left[z_{m k}\right]$ is the OF-matrix of size $M \times K$. Each element $z_{m k}$ defines the contribution of a source $m$ into a channel $k$. The SM described by (2) is schematically shown in figure 2a. The signals $\vec{s}$ of $M$ sources are mapped by the forward matrix $\mathbf{W}$ into the $L$ scalp potentials $\vec{p}$ which are mapped by the reference matrix $\mathbf{R}$ into the $K$ channels $\vec{c}$.

For the given locations of the sources and electrodes, $\mathbf{W}$ is fully determined by the VC and can be computed by solving the forward problem $[14,18]$. The matrix $\mathbf{R}$ depends on the choice of the reference, but it cannot be chosen arbitrarily. It must have certain properties to correspond to a physically realizable reference. For instance, $\mathbf{R}$ is singular and 


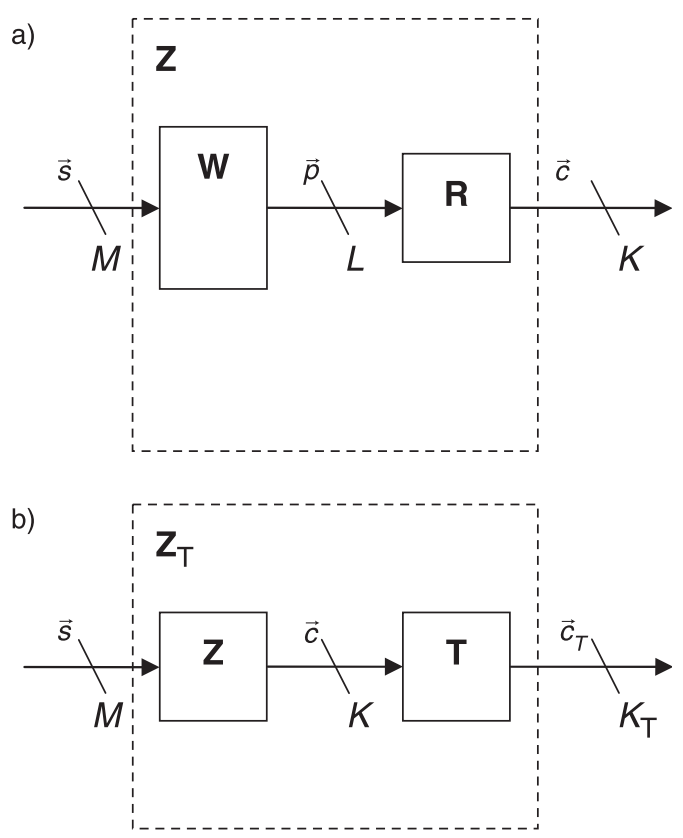

Figure 2. Schematic representation of the SM: (a) referencing procedure and (b) re-referencing procedure.

thus cannot be inverted. This is a fundamental limitation which reflects the fact that the potentials are relative quantities, and that at most $L-1$ independent channels can be obtained from $L$ electrodes.

The SM can be modified by a transformation of the channels $\vec{c}$, called the re-referencing procedure. This procedure is linear and thus can be characterized by a re-reference matrix. In figure $2 \mathrm{~b}$, a block diagram for the re-referencing procedure is shown, where $K$ recorded signals $\vec{c}$ are transformed into $K_{T}$ signals $\vec{c}_{T}$ by a re-reference matrix $\mathbf{T}$ of size $K \times K_{T}$. We denote the overall OF-matrix as $\mathbf{Z}_{\mathrm{T}}=\mathbf{W R T}=\mathbf{Z T}$. Contrary to $\mathbf{R}$, there are no constraints on $\mathbf{T}$ and it can be chosen arbitrarily.

It should be noted that $\mathbf{R}$ is typically non-square since some of the electrode signals are used as a reference. In this case, the number of electrodes $L$ is larger that the number of channels $K$. For simplicity, we assume $\mathbf{R}$ to be square with $K=L$, unless otherwise indicated. This assumption does not affect the results since one can ignore irrelevant channels.

\subsection{Performance measures}

Let us now introduce the performance measures used for the analysis of the OF-matrix and for comparisons of the references.

3.2.1 Relative power contribution (RPC). An important property of the SM is the "fusion" of the brain source signals in the EEG channels. This property determines the extent to which the sources can be resolved from the EEG, i.e. the extent to which they can be separated in different EEG channels. To assess this fusion, we use a performance measure called the RPC which describes the power contribution of local sources relative to the total power 
contribution of all the sources for an electrode. This power-based performance measure is used, since the source part of the model consists of stationary mutually uncorrelated noise sources. In terms of the OF-matrix $\mathbf{Z}$, the RPC for a channel $k$ can be expressed as:

$$
\operatorname{RPC}_{k}(r)=\frac{\sum_{m \in \Theta\left(d_{m k}<r\right)} z_{m k}^{2}}{\sum_{m=1}^{M} z_{m k}^{2}},
$$

where $d_{m k}$ is the maximum Euclidian distance between the dipoles and the orthogonal projection of the electrode onto the surface of the sphere where the dipoles are located, and $\Theta\left(d_{m k}<r\right)$ is a set of the dipoles located closer to the projection than the maximum distance $r$.

3.2.2 Cross-channel correlation coefficient (CCC). Interdependencies of the EEG channels recorded close to different cortical areas are often associated with functional connections of these areas. However, these interdependencies can be greatly affected by the $\mathrm{SM}$. This effect is analyzed in terms of the CCC. The CCC is relevant for the model because the model is linear, frequency independent, and has no time delays. Furthermore, the CCC is widely used for quantitative bivariate analysis of EEG channels $[8,19]$.

The correlation coefficient of two random variables $X$ and $Y$ measures the degree of linear interdependency between them. It is defined as the covariance of the variables normalized by the square root of the product of their individual variances: $\rho=\operatorname{cov}(X, Y) / \sqrt{\operatorname{var}(X) \operatorname{var}(Y)}$. It has range $[-1 \ldots 1]$ and vanishes for independent variables. For the model, the $\mathrm{CCC}$ for any pair of EEG channels $k$ and $l$ is fully determined by the OF-matrix $\mathbf{Z}$ according to the following formula:

$$
\operatorname{CCC}(k, l)=\frac{\sum_{i=1}^{M} z_{k i} z_{l i}}{\sqrt{\sum_{i=1}^{M} z_{k i}^{2} \sum_{i=1}^{M} z_{l i}^{2}}} .
$$

It should be noted that the performance measures defined by (3) and (4) are deterministic and depend only on $\mathbf{Z}$. This property makes the performance measures as well as the results independent of stochastic properties of the sources and thereby simplifies analysis of the SM.

\section{Localized reference}

As described in section 3, the SM can be modified by the choice of the reference. We may optimize the reference to minimize the impact of distant sources in each of the channels. Thus, a matrix $\mathbf{R}_{x}$ has to be found that minimizes this impact. The reference associated with $\mathbf{R}_{x}$ is called the LR, since it provides estimates of the activities generated in the neighborhood of the electrodes. The LR has optimal performance for the model.

We will proceed as follows. First, we will derive the LR in the form of a matrix $\mathbf{R}_{x}$ for a special case of the model with sources located exactly below the electrodes. Then, we will show that the LR has essentially the same matrix $\mathbf{R}_{x}$ for arbitrary source configurations. Finally, we will adapt the LR for re-referencing of EEGs recorded with common references and, thereby, make the LR practical. 
To this end, let us initially consider a hypothetical situation where there are only $M=L$ sources, denoted $\vec{s}_{x}$, located exactly below the $L$ electrodes. Here, $\mathbf{W}$ reduces to a square matrix $\mathbf{W}_{x}$ of size $L \times L$. Let us assume that $\mathbf{R}_{x}$ is square of size $L \times L$. Furthermore, we initially neglect the constraints for physically realizable references, i.e. we permit arbitrary matrices $\mathbf{R}_{x}$. In this case, equation (2) becomes

$$
\vec{c}=\vec{s}_{x} \mathbf{W}_{x} \mathbf{R}_{x} .
$$

Since the number of channels is equal to the number of sources one would ideally require that $\vec{c}=\vec{s}_{x}$, i.e. that each EEG channel is a recording of the signal of the source located below the electrode, and that the activity of the other $(L-1)$ sources is completely rejected. Using this requirement with equation (5) it follows that we should take

$$
\mathbf{R}_{x}=\mathbf{W}_{x}^{-1}
$$

where $\mathbf{W}_{x}^{-1}$ is the inverse of the forward matrix $\mathbf{W}_{x}$. In general, if $L$ is sufficiently small, then the columns and rows of $\mathbf{W}_{x}$ are linearly independent, the matrix has full rank $L$, and can be inverted. Thus, an ideal solution $\mathbf{R}_{x}$ can be obtained by (6).

We next justify that $\mathbf{R}_{x}$ optimized for the matrix $\mathbf{W}_{x}$ of size $L \times L$ is also essentially optimum for a matrix $\mathbf{W}$ of size $M \times L, M>L$. As before, we denote the sources located directly below the electrodes as $\vec{s}_{x}$. All the other sources are denoted $\vec{s}_{y}$. Now equation (2) becomes

$$
\vec{c}=\left(\vec{s}_{x} \mathbf{W}_{x}+\vec{s}_{y} \mathbf{W}_{y}\right) \mathbf{R},
$$

where $\mathbf{W}_{x}$ and $\mathbf{W}_{y}$ are the forward matrices for $\vec{s}_{x}$ and $\vec{s}_{y}$ respectively. This equation cannot be solved for the requirement $\vec{c}=\vec{s}_{x}$ since it is underdetermined. However, we may require $\vec{c}$ to be as close to $\vec{s}_{x}$ as possible in the Euclidian sense. Using (7), this requirement can be written as: $\left\|\vec{s}_{x}\left(\mathbf{W}_{x}-\mathbf{R}^{-1}\right)+\vec{s}_{y} \mathbf{W}_{y}\right\| \rightarrow$ min. Since $\vec{s}_{y}$ and $\vec{s}_{x}$ are independent, the most we can do is to minimize the term $\vec{s}_{x}\left(\mathbf{W}_{x}-\mathbf{R}^{-1}\right)$. In this case, $\mathbf{R}=\mathbf{W}_{x}^{-1}$, which is exactly the same matrix as in (6). Thus, the LR in the form of $\mathbf{R}_{x}$ is essentially optimum for the model with an arbitrary configuration of uncorrelated sources.

The matrix $\mathbf{R}_{x}$ has a full rank and thus it does not correspond to a physically realizable reference. In practice, the recordings are usually made with a common reference (see [6] for details about common references). Let us now modify the LR for re-referencing of EEGs recorded with a common electrode reference determined by $\mathbf{R}_{c}$. To this end, we consider the special case of the model (5) with an additional reference electrode, e.g. the M1. In this case, (5) becomes $\vec{c}=\vec{s}_{x} \mathbf{W}_{x}^{\prime} \mathbf{R}_{c}$, where the matrix $\mathbf{W}_{x}^{\prime}$ determines the mapping of $M$ sources $\vec{s}_{x}$ into $L=M+1$ scalp potentials. The reference electrode is used for the reference potential. Hence, $\mathbf{R}_{c}$ is of size $L \times M$ and $\mathbf{Z}=\mathbf{W}_{x}^{\prime} \mathbf{R}_{c}$ is square of size $M \times M$. The $L R$ can be computed in the form of a re-reference matrix $\mathbf{T}_{x}=\mathbf{Z}^{-1}$. For small $L$, the OF-matrix $\mathbf{Z}$ is non-singular and can be inverted. The re-reference matrix $\mathbf{T}_{x}$ applied to the channels referenced by the matrix $\mathbf{R}_{c}$ provides exactly the same $\mathrm{SM}$ as the matrix $\mathbf{R}_{x}$ applied to the potentials. For the sake of simplicity we will use $\mathbf{R}_{x}$ for comparison with other references in section 5 .

Another simple modification $\mathbf{R}_{x}^{\prime}$ can be made in order to make the LR completely independent of a common reference. The matrix $\mathbf{R}_{x}^{\prime}$ can be obtained by subtraction of the mean of each column from each element in the column of $\mathbf{R}_{x}: r_{i l}^{\prime}=r_{i l}-(1 / L) \sum_{i} r_{i l}$, where $r_{i l} \in \mathbf{R}_{x}, r_{i l}^{\prime} \in \mathbf{R}_{x}^{\prime}$, and $i \in\{1, \ldots, L\}, l \in\{1, \ldots, L\}$. Clearly, $\mathbf{R}_{x}^{\prime}$ suppresses the common 
component in the EEG channels including the common reference potential. Although $\mathbf{R}_{x}^{\prime}$ is not optimized, it has an effect which in terms of the performance measures is close to $\mathbf{R}_{x}$.

\section{Analysis of the $\mathrm{OF}$}

\subsection{Matrix representations of the references}

Any reference can be represented by the matrix $\mathbf{R}$. In figure $3 \mathrm{a}$, the matrices are schematically shown for different references. These matrices correspond to the 19 basic electrodes of the 10-20 electrode system and the reference electrodes M1 and M2.
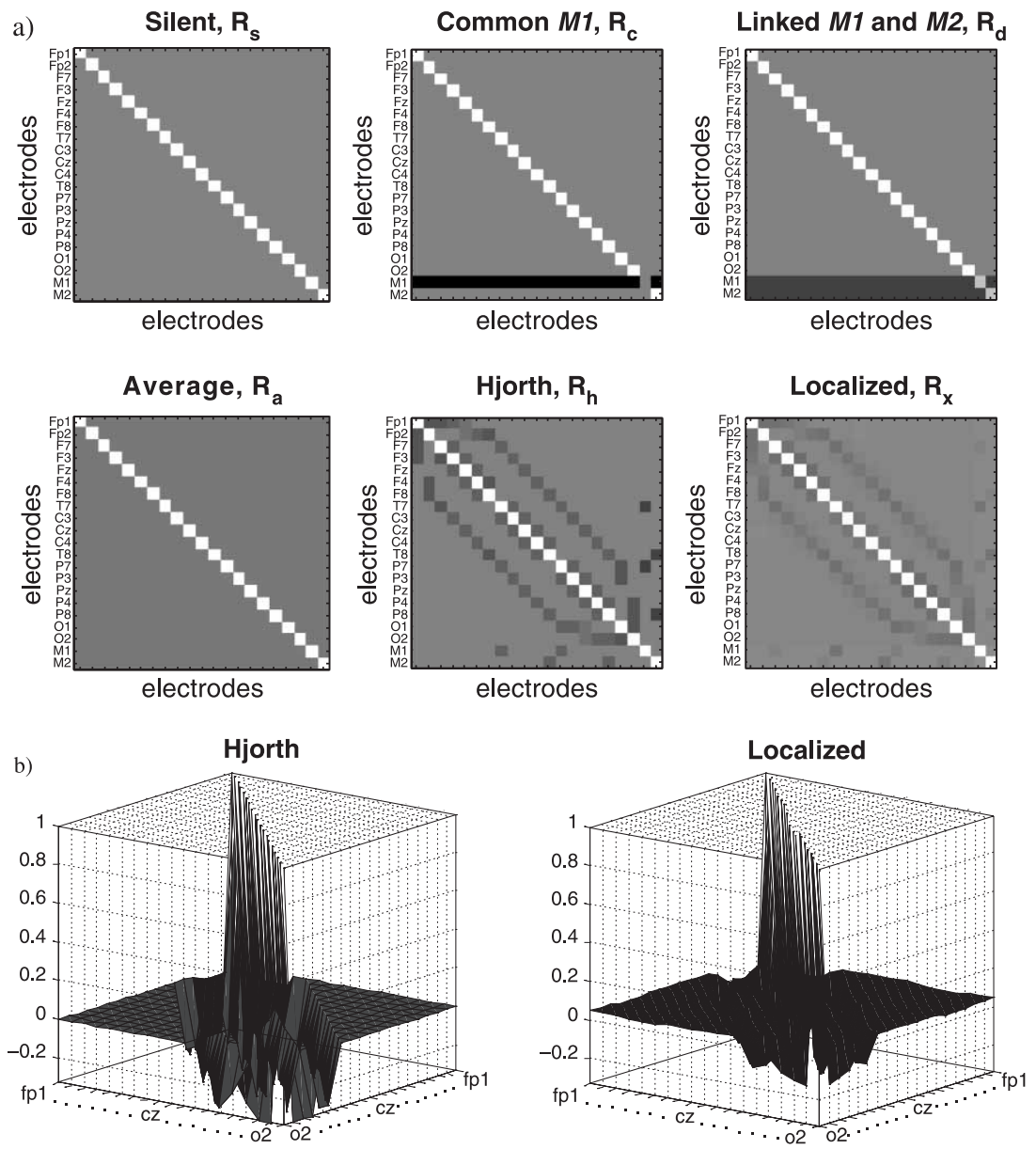

Figure 3. (a) Schematic representation of the square reference matrices. The matrix elements are shown in gray scale: the white color corresponds to 1 and the black to -1 . The matrix $\mathbf{R}_{x}$ is normalized to its maximum absolute value. Note, that the majority of matrix elements (light-gray color) for $\mathbf{R}_{a}$ and $\mathbf{R}_{x}$ have small, but different from zero values, whereas the light-gray color elements for the other matrices are zero; (b) The matrices $\mathbf{R}_{h}$ and $\mathbf{R}_{x}$ corresponding to the HR and the LR shown in 3-D space for the basic 19 electrodes of the standard 10-20 system. A larger number of neighbors of the recording electrodes have negative values in $\mathbf{R}_{x}$ compared to $\mathbf{R}_{h}$. 
5.1.1 Ideal silent reference and common references. We refer to detailed descriptions of the ideal silent reference and all the common references in [1,6]. Using these descriptions the matrices for these references can easily be constructed; see figure $3 \mathrm{a}$.

5.1.2 Hjorth laplacian reference (HR). The matrix $\mathbf{R}_{h}$ for the HR contains ones on its main diagonal. The elements of $\mathbf{R}_{h}$ corresponding to the nearest neighbors of the electrodes are equal to $-1 / n_{l}$, where $n_{l}$ is the total number of the nearest neighbors. All the other matrix elements are equal to zero. For the $F z$ and $P z$ electrodes, extra virtual neighbors are constructed as the equally weighted averages of $F p 1, F p 2$, and $O 1, O 2$ pairs as described by Hjorth [8]. Similar to the other realizable references, $\mathbf{R}_{h}$ is singular.

5.1.3 Localized reference (LR). The matrix $\mathbf{R}_{x}$ corresponding to the $\mathrm{LR}$ is found to be similar to $\mathbf{R}_{h}$. It contains the largest values on its main diagonal, and the nearest neighbors have the largest negative values. However, the values for non-nearest neighbor elements are negative as well. Furthermore, the elements for distant electrodes are positive with magnitudes in the range of 3-5\% of the maximal value in $\mathbf{R}_{x}$.

The matrices $\mathbf{R}_{x}$ and $\mathbf{R}_{h}$ are compared in figure $3 \mathrm{~b}$ (in this figure, M1 and M2 are excluded from the montage for illustration purposes). Although they are derived using fundamentally different approaches, i.e. the minimization of the impact of the distant sources in the SM $\left(\mathbf{R}_{x}\right)$ and the estimation of the radial current density through the skull $\left(\mathbf{R}_{h}\right)$, they are quite similar. Since the LR is optimized for the model, it can be seen as a refinement of the HR.

\subsection{OF and limits to spatial resolution}

Matrix representation of the SM enables us to assess limits to the spatial resolution for the EEG. Such an assessment is difficult to accomplish by other methods.

Assuming that the scalp potentials can be measured precisely, the error in the EEG channels is fully determined by small deviations of the mapping of the source signals into the scalp potentials. These deviations include small fluctuations of VC properties caused by, e.g., muscle contractions, blood flow, or changes of the temperature.

As explained in section 3.1, the VC is associated with the forward matrix W. Let us estimate how sensitive the potentials are to small perturbations of W. For simplicity, let us consider the situation with the sources located exactly under the electrodes, described by (5). Let the ideal potentials be $\vec{p}=\vec{s}_{x} \mathbf{W}_{x}$, and the measured potentials be $\vec{p}_{m}=\vec{s}_{x}\left(\mathbf{W}_{x}+\mathbf{E}\right)$, where $\mathbf{E}$ is some small perturbation of $\mathbf{W}_{x}$. Taking the difference we can write: $\vec{p}_{m}-\vec{p}=\vec{s}_{x} \mathbf{E}=\vec{p} \mathbf{W}_{x}^{-1} \mathbf{E}$, and hence, $\left\|\vec{p}_{m}-\vec{p}\right\|=\left\|\vec{p} \mathbf{W}_{x}^{-1} \mathbf{E}\right\| \leq\|\vec{p}\|\left\|W_{x}^{-1}\right\|\|\mathbf{E}\|$, which is equivalent to $\left(\left\|\vec{p}_{m}-\vec{p}\right\| /\|\vec{p}\|\right) \leq\left\|\mathbf{W}_{x}^{-1}\right\|\left\|\mathbf{W}_{x}\right\|\left(\|\mathbf{E}\| /\left\|\mathbf{W}_{x}\right\|\right)$. Finally, we can write:

$$
\frac{\left\|\vec{p}_{m}-\vec{p}\right\|}{\|\vec{p}\|} \leq k\left(\mathbf{W}_{x}\right) \frac{\|\mathbf{E}\|}{\left\|\mathbf{W}_{x}\right\|}
$$

where $k\left(\mathbf{W}_{x}\right)=\left\|\mathbf{W}_{x}^{-1}\right\|\left\|\mathbf{W}_{x}\right\|$ is the condition number of the forward matrix $\mathbf{W}_{x}$. For $l_{2}$-norm, it can be shown that $k\left(\mathbf{W}_{x}\right)=\sigma_{\max }\left(\mathbf{W}_{x}\right) / \sigma_{\min }\left(\mathbf{W}_{x}\right)$, where $\sigma_{\max }\left(\mathbf{W}_{x}\right)$ and $\sigma_{\min }\left(\mathbf{W}_{x}\right)$ are the largest and smallest singular values [20].

Thus, the relative error in the potentials e $(\vec{p})=\left\|\vec{p}_{m}-\vec{p}\right\| /\|\vec{p}\|$ is bounded by $k$ times the relative error in the forward matrix $e\left(\mathbf{W}_{x}\right)=\|\mathbf{E}\| /\left\|\mathbf{W}_{x}\right\|$. Furthermore, in the absence of measurement noise $\mathrm{e}(\vec{p})=\mathrm{e}(\vec{c})=\left\|\vec{c}_{m}-\vec{c}\right\| /\|\vec{c}\|$. 
The condition number $k$ is shown in figure 4 for seven different electrode arrays. The electrodes are located on the surface of the scalp exactly above the sources uniformly distributed in the cortical layer. Each array is characterized by a number of electrodes $L \in\{8,51,104,144,212,259,314\}$ and a corresponding surface interelectrode distance $d(L) \in\{9.1,3.6,2.5,2.2,1.8,1.6,1.5\} \mathrm{cm}$. The condition number for the corresponding matrices $\mathbf{W}_{x}(L)$ is $k\left(\mathbf{W}_{x}(L)\right) \in\{1.8,8.6,28.1,53.2,118.8,188.4,303.9\}$. For the basic 19 electrodes of the standard 10-20 electrode system, $k=2.8$. It can be seen from figure 4 that $k$ increases rapidly with $L$, thus the error $e(\vec{c})$ becomes substantial for larger $L$.

Figure 4 can be associated with limits to the spatial resolution for the scalp EEG. As an example, let us assume that the largest relative error acceptable for the EEG channels is $\mathrm{e}(\vec{c})=0.5$. We assume that the perturbations of the $\mathrm{VC}$ are small and lead to the relative error e $\left(\mathbf{W}_{\mathrm{x}}\right)=0.01$. Then, the limiting value of $k$ can be estimated at 50. It corresponds to an electrode array with about 140 electrodes, with an interelectrode distance of about $2 \mathrm{~cm}$. Hence, distances down to $2 \mathrm{~cm}$ can be resolved from the EEG.

An impact of measurement noise can be analyzed similarly. It can also be shown that $e(\mathbf{C})$ increases rapidly with $L$. This is consistent with our conclusion that EEG channels for dense electrode arrays may have substantial errors.

\subsection{Impact of the number of sources}

The amount of macrocolumns in the human cortex can be estimated to be about $10^{4}$. Nevertheless, the model is only a crude approximation of the EEG recording procedure, and therefore the number of the equivalent dipoles may be reduced without significant changes of the model accuracy. This simplifies the model, and makes it easier to utilize.

The sensitivity of the model to the number of dipoles is analyzed in terms of the performance measures RPC and CCC defined by (3) and (4) respectively. By way of illustration the ideal silent reference is used. Results for the other references are similar.

In figure 5 the RPC is shown for the ideal silent reference and for three different arrays of dipoles denoted as $D_{1}, D_{2}$ and $D_{3}$ the arrays have respectively 104,1052 and 10,852 uniformly distributed dipoles with corresponding densities $0.22,2.19$, and

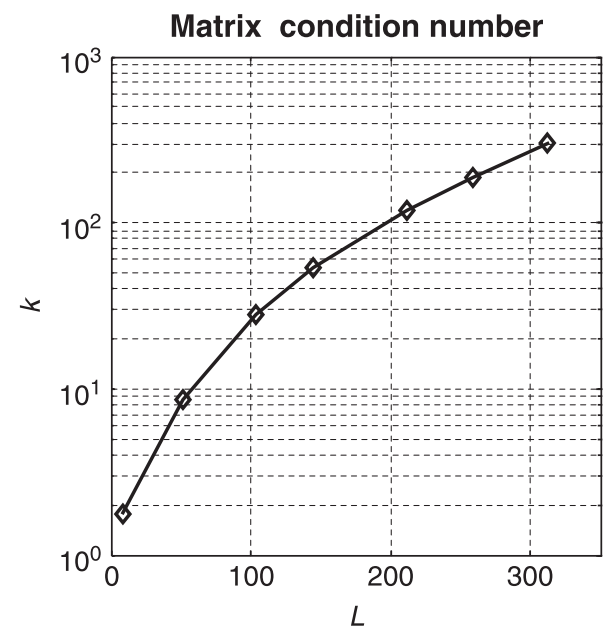

Figure 4. Condition number $k\left(\mathbf{W}_{x}\right)$ is plotted vs. the total number of electrodes $L$. 


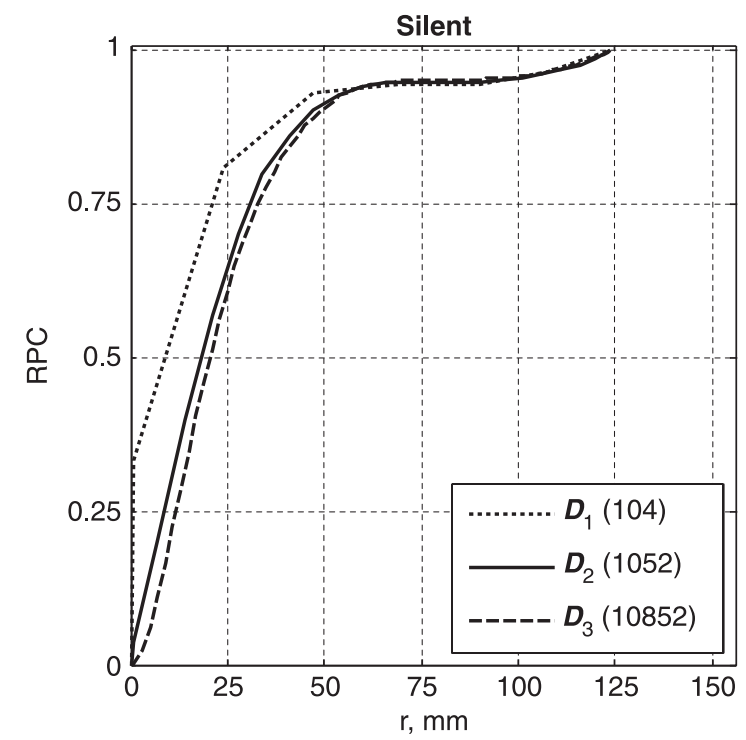

Figure 5. The RPC computed for different dipole configurations of the model with the ideal silent reference. The RPC at the electrode position $C z$ is shown for three source configurations having 104, 1052 and 10,852 dipoles.

22.55 dipoles $/ \mathrm{cm}^{2}$. It can be seen from figure 5 that $D_{1}$ provides inaccurate approximation of $D_{3}$ in terms of the RPC. However, $D_{2}$ and $D_{3}$ provide quite similar results. The difference between them is below the assumed accuracy of the model (10-20\%). The same three configurations of the model are analyzed in terms of the CCC. No significant differences are found across the arrays. We conclude that for the performance measures the number of dipoles in the model can be reduced to those of $D_{2}$ without significant changes in the performance. Therefore, $D_{2}$ is utilized for the model.

\section{4 $\mathrm{OF}$ and comparison of the references}

The OF-matrix determines the SM during recording of the EEG. The OF-matrix is analyzed in terms of the RPC and the CCC defined by (3) and (4) respectively. The source part of the model involves 1052 radial dipoles located in the cortical layer as described in section 2.2. The montage involves the 19 basic electrodes (M1 and M2 were removed after the referencing procedure).

5.4.1 Relative power contribution (RPC). In figure 6, the RPC is plotted for different radiuses of the local area $r$ and for all the references. The RPC is computed at the electrode positions $C z$ and $P 8$. These positions are in the middle and on the border of the electrode array respectively, and thus correspond to the two extremes.

The results suggest that less than $10-20 \%$ of the total power contribution originates from a local area of $5 \mathrm{~mm}$ radius (the size of a typical electrode cup). It can be seen from figure 6 that the LR has the best localization performance compared to the other references, i.e. the RPC curve is steepest. At $50 \mathrm{~mm}$ (the typical interelectrode distance for the standard 10-20 system) the RPC for the LR has approximately $95 \%$ of its maximum value, which is larger than approximately the $90 \%$ for the HR, and much larger than for all the other 

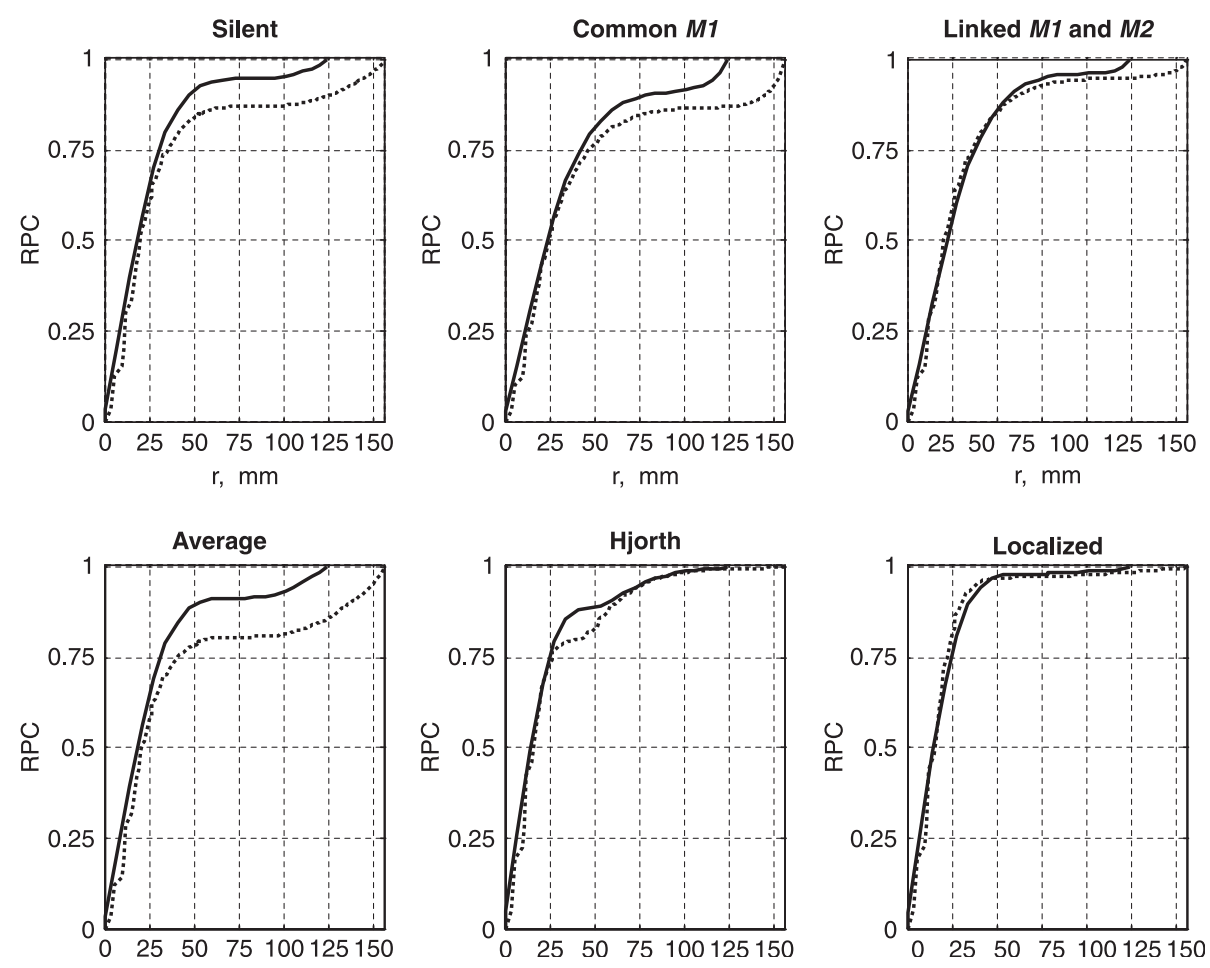

Figure 6. The RPC computed at the electrode positions $C z$ (solid line) and $P 8$ (dotted line) is shown vs. the radius of the local area $r$ for different references. See the text for further explanations.

references. The LR also provides the most similar RPC curves for both of the electrodes $C z$ and $P 8$, which means that the performance of the LR is less dependent on boundary effects.

5.4.2 Cross-channel correlation coefficient (CCC). Figure 7 shows the dependence of the $\mathrm{CCC}$ on the interelectrode distances. The CCC is computed for all the pairs of the electrodes and plotted in the figure in dots.

Figure 7 is first described for the ideal silent reference, and then the effects of all the references are compared. The figure for the silent reference describes the effect of the VC only.

The channels corresponding to closely spaced electrodes may have a substantial CCC due to adjacent sources. Less expected is substantial negative CCC for distantly located electrodes. This can be explained by the anti-symmetric property of the dipole-field. The field induced by a dipolar source at a neighboring electrode is also induced, with a reversed sign and damped amplitude, at diametrically opposite electrodes. Thus, electrodes pick up similar potentials of different polarity, and therefore the corresponding EEG channels are linearly interdependent and have substantial negative CCC. The electrodes separated by intermediate distances of about $100 \mathrm{~mm}$ are least affected by the VC. The dipoles located under one of such electrodes are oriented nearly perpendicular to those under the other. Hence, the electrode potentials are practically independent and have a small CCC.

It can be seen from figure 7 that the common electrode reference is characterized by a large deviation of the CCC. This can be explained by dependence of the CCC on the distances 

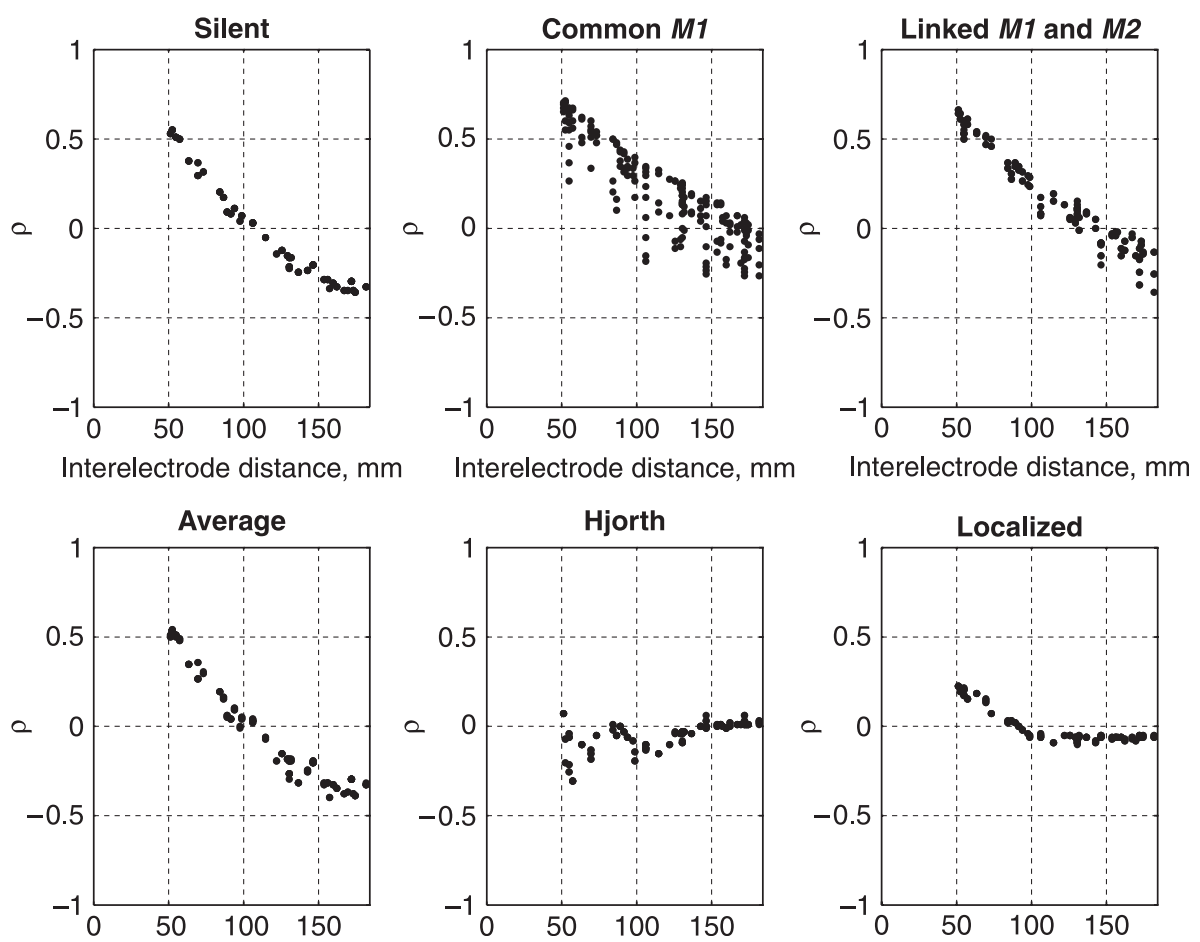

Figure 7. The CCCs are shown vs. the interelectrode distances for different references. See the text for further explanations.

of the electrodes from the reference electrode. The common digitally linked mastoids reference is a considerable refinement of the common electrode since it provides smaller deviation of the CCC, and better approaches the ideal silent reference. The common average reference is the nearest approximation of the ideal silent reference. However, the common average reference can be expected to provide results similar to the ideal silent reference only when the number of electrodes is large and is not recommended for the 10-20 system. This result follows from the fact that the surface integral of potential over a closed surface with internal current sources is zero; a large number of surface electrodes approximates this surface integral better [21].

The ideal silent reference as well as all the common references provides inaccurate approximations of the signals induced by the sources located under the electrodes. The HR and the LR are different in this respect. Both are characterized by small CCCs for distant electrodes. The LR provides less dispersion of CCCs than the HR and is almost ideal for the electrodes located further than two interelectrode distances from each other (about $100 \mathrm{~mm}$ ).

\section{Discussion}

The SM, i.e. the mapping of brain source signals into EEG channels, seems under-exposed and may cause misinterpretations of the EEG. This paper analyzes the SM using its matrix form representation for a generation model for the desynchronized spontaneous EEG and for 
five references commonly used in hospitals. The results show that the HR is the most suitable among the studied existing references for estimation of the localized cortical activity.

A novel reference is developed using a model-based optimization of the SM. This reference minimizes the activities of the cortical areas distant from the electrodes, thereby providing "focused" estimates of the activities under the electrodes. Therefore, this reference is called the LR. Given the model, the LR has a better performance compared to the existing references, and is the most similar to the HR.

Given the model, the LR is an optimum estimator of local cortical activity. However, the difference with the HR is moderate compared to the overall accuracy of the model. Hence, for the head of a given person, it is unlikely that the LR optimized with the parameters of an average head has remarkably better performance than the HR. Therefore, the HR seems a good choice for estimation of cortical activities when the parameters of a head are a priori unknown.

The LR, however, can be tuned to a particular head using a (more realistic) model with measured parameters. The LR seems particularly suitable for combined EEG-MRI studies because MRI scans can be used for realistic models [22]. It is important to note that since the LR is an optimum estimator of cortical activity, it can be used in theoretical studies for comparison purposes. For instance, it would be interesting to compare the performance of the LR with surface Laplacians that are also model dependent and have slightly better performance than the HR $[9,10]$.

The LR is proposed for an estimation of the activities of the local cortical areas. A possible application is an assessment of the statistical relationship between signals generated at different brain sites that can be used to characterize functional connectivity of the brain [23]. For this application, even small gains in crosstalk reduction in the SM may be important.

The LR involves the entire electrode array. It can be easily adapted for unusual arrays which may be inappropriate for the HR (the HR requires equidistantly spaced neighboring electrodes).

The LR involves all the recording electrodes from the array. This property may promote dispersion of prominent artifacts or noise in a single channel across all the channels. In fact, the absolute values of the elements in the reference matrix $\mathbf{R}_{x}$ can be associated with "weights" of each of the electrode signal in the channel signals, i.e. $\mathbf{R}_{x}$ directly characterizes the dispersion effect (see also figure 3). For the LR, this dispersion can be better understood through comparison to the HR. The HR suppresses the common component of the electrode potentials and disperses the activity among the nearest neighbor electrodes. The LR has a similar effect, but it does not reduce the common component completely, affects more neighbors, and has some minor dispersion effect on all the electrodes. For instance, $50 / 60 \mathrm{~Hz}$ noise may be substantially reduced by the LR if it contributes (approximately) equally to all the electrodes, but a movement artifact of a single recording electrode will be dispersed mainly through the nearest neighbors and much less through the other electrodes.

The LR is optimized for the model with mutually uncorrelated sources, thus for the desynchronized EEG. The performance of the LR may decrease for other types of the EEG, e.g. recordings of pathological brain discharges during generalized epileptic seizures. In this case, the LR may provide inaccurate estimation of the activity of the local areas. For such recordings, the performance can be improved only through optimization of the reference for a particular correlation pattern. In other words the LR is essentially optimum for the case when a priori knowledge about head geometry is available, but not about the sources.

The volume conduction part of the model uses classical conductivity values described in [11]. However, recent publications suggest that the brain-to-skull conductivity ratio 
is significally smaller than the classical one [13]. We compare model performances for the classical and for the recently found conductivity values in terms of the CCC and the RPC measures. The results show that the difference is at most $5 \%$ for the RPC and even smaller for the CCC. Thus, we conclude that the model does not depend critically on the conductivity values and that the results obtained for the classical values can be used as a fair approximation for the other values.

The analysis of the SM is done for the standard 10-20 electrode system since this system is considered sufficient for most clinical cases and is widely used in hospitals. Although the SM depends on the number of electrodes, the analysis for larger electrode arrays reveals qualitatively similar results and thus the main conclusions remain the same as for the 10-20 system.

The matrix representation of the SM allows us to assess limits to the spatial resolution for the EEG. The resolution is found to depend on the condition number of the forward matrix. Small deviations in the VC caused by physiological processes in the head are effectively amplified by the condition number. A crude estimate is that distances down to $2 \mathrm{~cm}$ can be resolved from the EEG. This corresponds to arrays of about 140 electrodes. This estimate is consistent with other estimates $[24,25]$ obtained experimentally.

\section{References}

[1] Niedermeyer, E. and Lopes da Silva, F., 1999, Electroencephalography: Basic Principles, Clinical Applications, and Related Fields, 4th ed. (Philadelphia: Lippincott Williams and Wilkins), Ch. 5.

[2] Lopes da Silva, F., 2004, Functional localization of brain sources using EEG and/or MEG data: volume conductor and source models, Magnetic Resonance Imaging, December, 22(10), 1533-1538.

[3] Nunez, P.L., 1995, Neocortical Dynamics and Human EEG Rhythms (New York: Oxford University Press), Ch. 1-2.

[4] Malmivuo, J., Suihko, V. and Eskola, H., 1997, Sensitivity distributions of EEG and MEG measurements, IEEE Transactions on Bio-Medical Engineering, March, 44(3), 196-208.

[5] Michel, C.M., Murray, M.M., Lantz, G., Gonzalez, S., Spinelli, L. and Grave de Peralta, R., 2004, EEG source imaging, Clinical Neurophysiology, October, 115(10), 2195-2222.

[6] Nunez, P.L., Srinivasan, R., Westdorp, A.F., Wijesinghe, R.S., Tucker, D.M. and Silberstein, R.B., 1997, EEG coherency. I: statistics, reference electrode, volume conduction, Laplacians, cortical imaging, and interpretation at multiple scales, Electroencephalography and Clinical Neurophysiology, November, 103(5), 499-515.

[7] Srinivasan, R., Nunez, P.L. and Silberstein, R.B., 1998, Spatial filtering and neocortical dynamics: estimates of EEG coherence, IEEE Transactions on Bio-Medical Engineering, July, 45(7), 814-826.

[8] Hjorth, B., 1975, An on-line transformation of EEG scalp potentials into orthogonal source derivations, Electroencephalography and Clinical Neurophysiology, November, 39(5), 526-530.

[9] Perrin, F., Pernier, J., Bertrand, O. and Echallier, J.F., 1989, Spherical splines for scalp potential and current density mapping, Electroencephalography and Clinical Neurophysiology, February, 72(2), 184-187.

[10] He, B., Lian, J. and Li, G., 2001, High-resolution EEG: a new realistic geometry spline Laplacian estimation technique, Clinical Neurophysiology, May, 112(5), 845-852.

[11] Rush, S. and Driscoll, D.A., 1969, EEG electrode sensitivity-an application of reciprocity, IEEE Transactions on Bio-Medical Engineering, January, 16(1), 15-22.

[12] Gutierrez, D., Nehorai, A. and Muravchik, C.H., 2004, Estimating brain conductivities and dipole source signals with EEG arrays, IEEE Transactions on Bio-Medical Engineering, December, 51(12), 2113-2122.

[13] Zhang, Y., van Drongelen, W. and He, B., 2006, Estimation of in vivo human brain-to-skull conductivity ratio with the aid of intracranial electrical stimulation, Applied Physics Letters, November, 89, 223903.

[14] Giapalaki, S.N. and Kariotou, F., 2006, The complete ellipsoidal shell-model in EEG imaging, Abstract and Applied Analysis, Article ID 57429.

[15] Yan, A.M., Marechal, E. and Nguyen-Dang, H., 2001, A finite-element model of mixed-mode delamination in laminated composites with an R-curve effect, Composites Science and Technology, August, 61(10), $1413-1427$.

[16] Nunez, P.L., Silberstein, R.B., Shi, Z., Carpenter, M.R., Srinivasan, R., Tucker, D.M., et al., 1999, EEG coherency II: experimental comparisons of multiple measures, Clinical Neurophysiology, March, 110(3), 469-486. 
[17] Towle, V.L., et al., 1993, The spatial location of EEG electrodes: locating the best-fitting sphere relative to cortical anatomy, Electroencephalography and Clinical Neurophysiology, January, 86(1), 1-6.

[18] Kavanagh, R.N., Darcey, T.M., Lehmann, D. and Fender, D.H., 1978, Evaluation of methods for threedimensional localization of electrical sources in the human brain, IEEE Transactions on Bio-Medical Engineering, September, 25(5), 421-429.

[19] Quian Quiroga, R., Kraskov, A., Kreuz, T. and Grassberger, P., 2002, Performance of different synchronization measures in real data: a case study on electroencephalographic signals, Physical Review. E, Statistical, Nonlinear, and Soft Matter Physics, April, 65(4 Pt 1), 41903.

[20] Moon, T.K. and Stirling, W.C., 1999, Mathematical Methods and Algorithms for Signal Processing, Bk\&CD Rom ed. (Englewood Cliffs, NJ: Prentice Hall), Ch. 4.

[21] Nunez, P.L. and Srinivasan, R., 2006, A theoretical basis for standing and traveling brain waves measured with human EEG with implications for an integrated consciousness, Clinical Neurophysiology, November, 117(11), $2424-2435$.

[22] Tizzard, A., Horesh, L., Yerworth, R.J., Holder, D.S. and Bayford, R.H., 2005, Generating accurate finite element meshes for the forward model of the human head in EIT, Physiological Measurement, April, 26(2), S251-S261.

[23] Lee, L., Harrison, L.M. and Mechelli, A., 2003, A report of the functional connectivity workshop, dusseldorf 2002, Neuroimage, June, 19(2 Pt 1), 457-465.

[24] Ryynanen, O.R.M., Hyttinen, J.A.K., Laarne, P.H. and Malmivuo, J.A., 2004, Effect of electrode density and measurement noise on the spatial resolution of cortical potential distribution, IEEE Transactions on BioMedical Engineering, September, 51(9), 1547-1554.

[25] Gevins, A., Le, J., Brickett, P., Reutter, B. and Desmond, J., 1991, Seeing through the skull: advanced EEGs use MRIs to accurately measure cortical activity from the scalp, Brain Topography, 4(2), 125-131. 


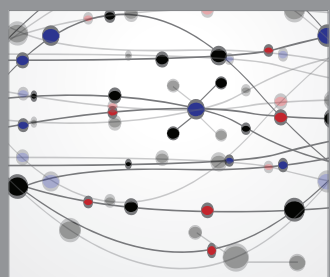

The Scientific World Journal
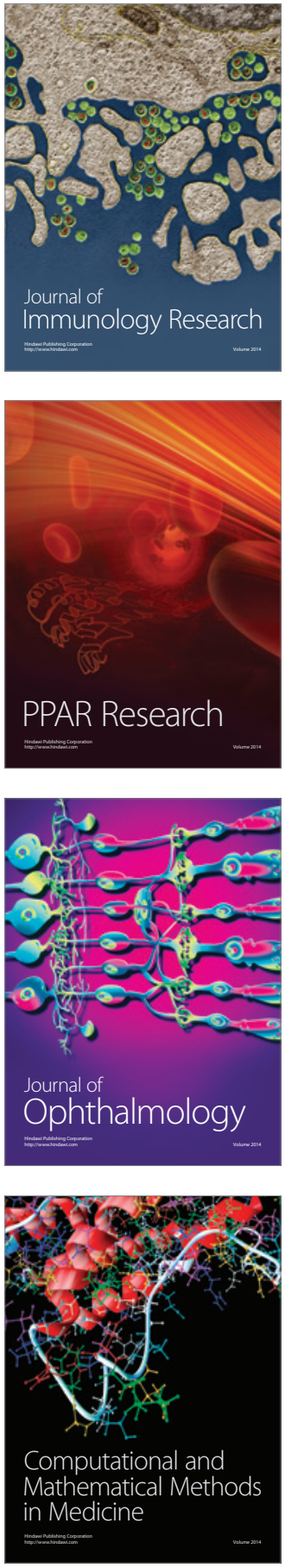

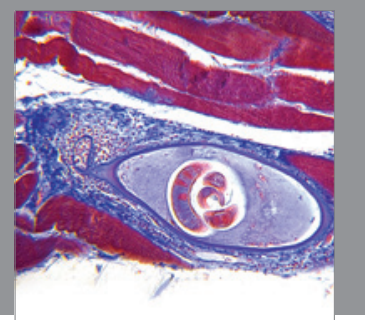

Gastroenterology

Research and Practice
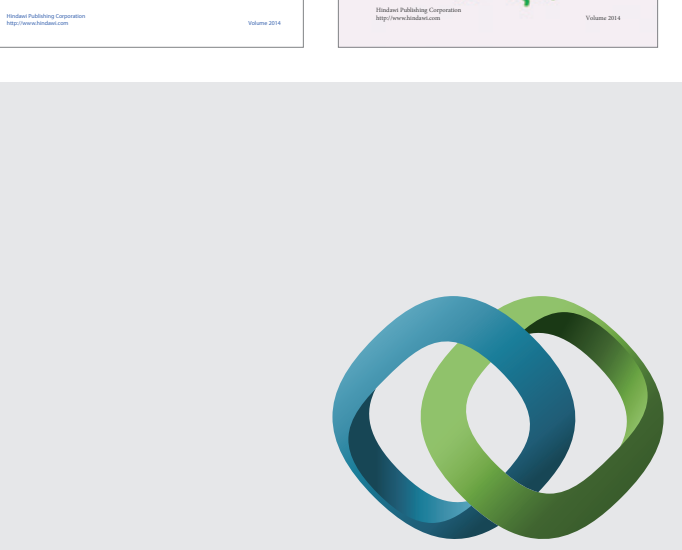

\section{Hindawi}

Submit your manuscripts at

http://www.hindawi.com
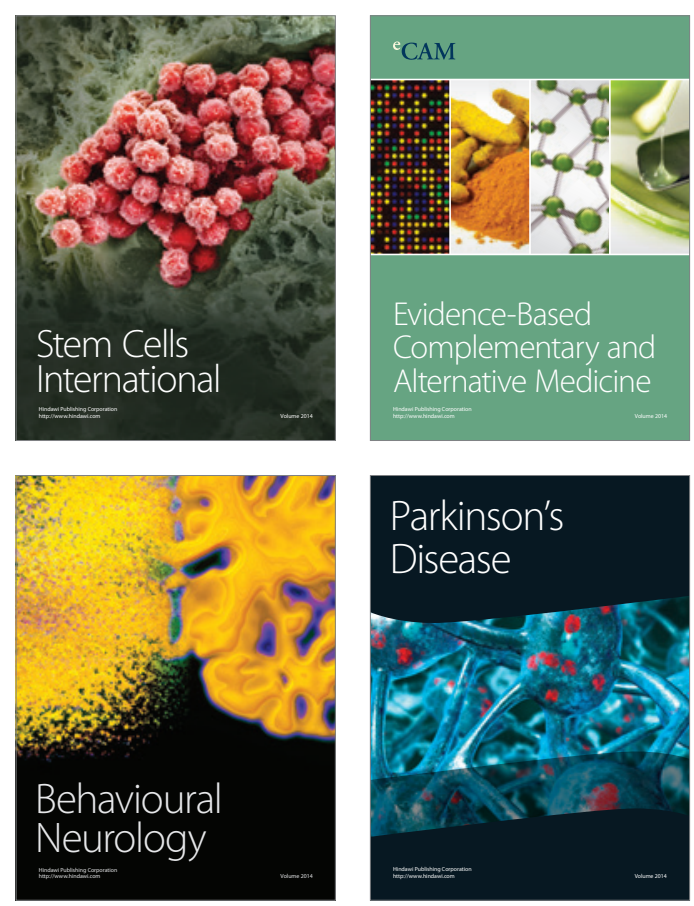

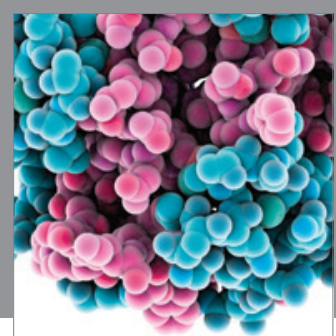

Journal of
Diabetes Research

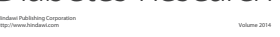

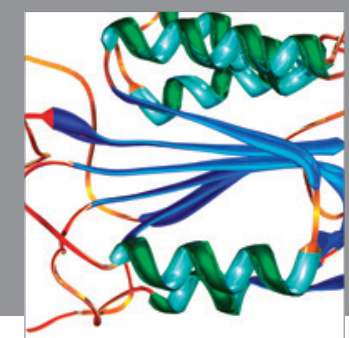

Disease Markers
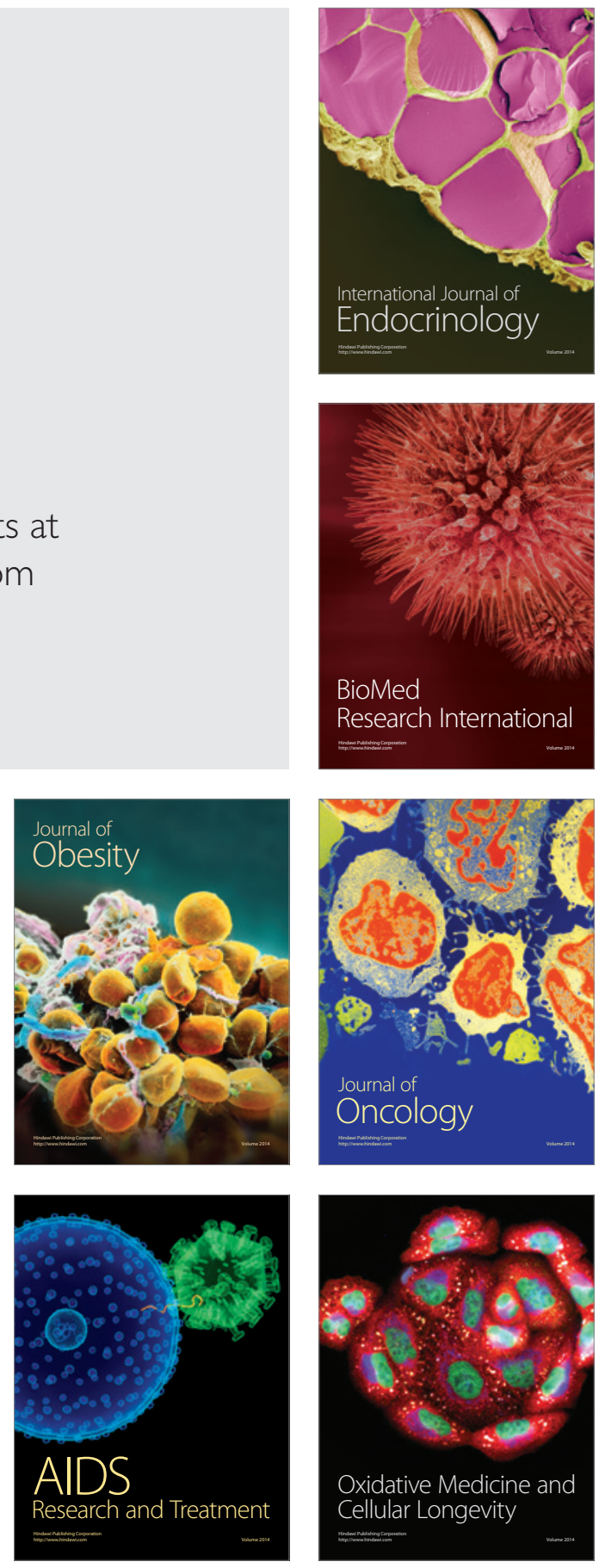